\title{
Transverse Shear Effects in Bimodular Composite Laminates
}

\author{
C. W. BERT AND F. GORDANINEJAD \\ School of Aerospace Mechanical, and Nuclear Engineering \\ The University of Oklahoma \\ Norman, OK 73019
}

\begin{abstract}
A closed-form solution for the Timoshenko-type shear correction coefficient $\left(\mathrm{K}^{2}\right)$ governing the deflection of bimodular composite laminates in cylindrical bending is presented. The bendingstress distribution for a laminate constructed of bimodular materials (which have different elastic moduli in tension and compression) is used in the (wo-dimensional equilibrium equation to obtain the transverse shear-stress distribution. This shear-stress distribution is used to obtain expressions for the shear correction coefficient (based on equivalent shear strain energy) and the maximum dimensionless transverse shear stress $\left(\tau_{x z}\right)_{\max }$. Finally, the effects of the elastic-constant ratios on the neutral-surface location and shear correction coefficient for laminates consisting of either unimodular or bimodular materials are studied.
\end{abstract}

\section{INTRODUCTION}

$\mathbf{M}$ ATERIALS WHICH HAVE DIFFERENT MODULI IN TENSION AND IN COMpression are called bimodular materials. Rock, concrete, cord-rubber, paperboard, and certain biological tissues are examples of such materials. Even aramid-fiber, polymer-matrix composites exhibit some bimodularity. The analysis of laminated bimodular material is more complicated than unimodular material (ordinary material) due to the dependency of the material stiffness on the material properties, which indeed depend on the state of stress (i.e., tensile or compressive) in the laminate.

Although transverse shear deformations have been considered in the analyses of bimodular laminates [1-4] in recent years, there has been no effort to include the effect of bimodularity of the material on the Timoshenkotype shear correction coefficient, $\mathrm{K}^{2}$. In other works, it has been tacitly assumed that the value of $\mathrm{K}^{2}$ is the same as that of an ordinary-material laminate. The use of such a shear correction factor in predicting the deflection of shear deformable, ordinary-material laminates in cylindrical bending is well established [5,6]. It is noted that Whitney [5] extended Chow's symmetric-laminate work [7] to arbitrary laminates. Also, it can be shown that the resulting expressions in $[5,6]$ are algebraically equivalent.

In this paper, a straight-forward approach analogous to that used in elementary shear theory for single-layer ordinary materials is employed. It 
may be considered to be a generalization of the work of Bert [6] from ordinary-material laminates to bimodular-material laminates.

\section{THEORY AND FORMULATION}

Consider a rectangular-cross-section laminated beam of thickness $h$. The origin of the Cartesian coordinate system is located on the mid-surface of the beam with the $x$ axis being along the length of the beam and the $z$ axis being measured positive downward. The same displacement field as used in shear deformable beam theory is implemented here:

$$
\begin{aligned}
& u(x, z)=u^{\circ}(x)+z \psi(x) \\
& w(x, z)=w^{\circ}(x)
\end{aligned}
$$

The axial normal strain and transverse shear strain are given by

$$
\varepsilon_{x}=u_{, x}^{\circ}+z \psi, x ; \gamma_{x z}=\psi+w_{, x}^{\circ}
$$

where a comma denotes the derivative with respect to the quantity following it. The longitudinal bending stress at any distance $z$ from the midplane of the laminate is

$$
o_{x}=E^{(k)} \varepsilon_{x} \quad k=c, t
$$

Also

$$
\tau_{x z}=G^{(k)} \gamma_{x z}
$$

The longitudinal stress resultant and stress couple are defined as

$$
(N, M)=\int_{-h / 2}^{h / 2}(1, z) \sigma_{x} d z
$$

With the aid of Equations (2), (3), and (5), one can derive the following laminate constitutive relation

$$
\left\{\begin{array}{l}
N \\
M
\end{array}\right\}=\left[\begin{array}{ll}
A & B \\
B & D
\end{array}\right]\left\{\begin{array}{l}
u_{, x}^{\circ} \\
\psi, x
\end{array}\right\}
$$

where

$$
(A, B, D)=\int_{-h / 2}^{h / 2} E^{(k)}\left(1 z, z^{2}\right) d z \quad k=c, t
$$


In the absence of body force, the two-dimensional static equilibrium equation for forces in the direction along the length of the beam is

$$
\sigma_{x, x}+\tau_{x z, z}=0
$$

Integrating Equation (7) with respect to $z$ and using Equations (3) and (4), one has

$$
\gamma_{x z}=-\left[G^{(k)}\left(A D-B^{2}\right)\right]^{-1}\left[(D a-B b) N_{, x}+(A b-B a) M, x\right]
$$

where $a$ and $b$ are "partial stiffnesses" for stretching and bending-stretching coupling defined by the following expressions for the convex bending case (top layers in compression and bottom layers in tension)

$$
(a, b)= \begin{cases}\int_{-h / 2}^{z}(1, z) E^{c} d z & z_{n}>z>-h / 2 \\ \int_{h / 2}^{z_{n}}(1, z) E^{c} d z+\int_{z_{n}}^{z}(1, z) E^{l} d z & h / 2>z>z_{n}\end{cases}
$$

Multiplying Equation (7) by unity and $z$, respectively, and then integrating through the thickness, one obtains the following equilibrium equations, which coincide with those of elementary beam theory,

$$
M_{, x}=Q, \quad N_{, x}=0
$$

where

$$
Q=\bar{\gamma}_{x z} \int_{-h / 2}^{h / 2} G^{(k)} d z
$$

Therefore, Equation (8) simplifies as follows:

$$
\gamma_{x z}=-Q(A b-B a)\left[G^{(k)}\left(A D-B^{2}\right)\right]^{-1}
$$

Extending the definition of the shear correction coefficient as used in [6], which is based on the shear strain energy, to the case of bimodular material, one has

$$
K^{2}=\frac{\left(A D-B^{2}\right)^{2}}{\left.I \int_{-h / 2}^{h / 2} G^{(k)} d z J \iint_{-h / 2}^{h / 2}\left(I / G^{(k)}\right)(A b-B a)^{2} d z\right]}
$$


If $G$ is constant throughout the thickness of the beam, Equation (13) can be simplified to

$$
K^{2}=\frac{\left(A D-B^{2}\right)^{2}}{h \int_{-h / 2}^{h / 2}(A b-B a)^{2} d z}
$$

By changing the coordinate from $z$ measured from the midplane to $z^{\prime}$ measured from the neutral-surface position, one can simplify Equation (14) as follows:

$$
K^{2}=\frac{D^{2}}{h \int_{-h_{c}}^{h_{t}} b^{2} d z^{\prime}}
$$

To evaluate the stiffnesses $(A, B$, and $D)$, one needs to know the location of the neutral-surface position. A closed-form solution can be obtained only when the neutral-surface position $\left(z_{n}\right)$ and thus, the stiffnesses do not depend on $x$. The same criterion to define $z_{n}$ as used in [4] is applied here

$$
\varepsilon_{x}=0
$$

Using Equations (2) and (5) and assuming* that

$$
N=0
$$

one gets

$$
z_{n}=B / A=\text { constant }
$$

Equation (18) can be solved explicitly for $z_{n}$.

It is convenient to introduce the dimensionless transverse shear stress defined as follows:

$$
\bar{\tau}_{x z}=\frac{\tau_{x z}}{Q / h}
$$

Combining Equations (4) and (12) and measuring $z^{\prime}$ from the neutralsurface location, one obtains

$$
\tau_{x z}=b h / D
$$

Note that this assumption is made only for the bimodular case (not unimodular). 
Detailed derivations for $\mathrm{K}^{2}$ and $\left(\bar{\tau}_{x z) \max }\right.$ are given in the Appendix.

\section{NUMERICAL RESULTS}

Three cases of laminates constructed of bimodular materials are considered here: single-layer, two-layer, and three-layer. Numerical results obtained from closed-form solutions for the shear correction coefficient and the maximum dimensionless shear stress for different bimodular-material parameters are presented. Also, the effects of elastic-constant ratios on neutral-surface position and $\mathrm{K}^{2}$ for both unimodular and bimodular laminates (up to three layers) are studied. The laminates are assumed to be made of equal-thickness layers and the effect of the sign of the longitudinal normal strain on shear moduli is ignored (that is, $G^{c} / G^{\prime}=1$ ).

In the case of single-layer bimodular material (see the Appendix), it is interesting to note that both the transverse shear correction coefficient and the maximum transverse shear stress are unaffected by the bimodular ratio $E^{c} / E^{t}$. However, this does not imply that the distribution of shear stress is the same for unimodular and bimodular materials (see Fig. A-1). The effect of bimodular ratio on the location of the neutral surface is studied in Table 1 . For values of $E^{c}<E^{t}$ (e.g., aramid-cord/rubber), $h_{t}$ falls within the lower half of the thickness; whereas for materials such as rock $\left(E^{c}>E^{t}\right), h_{t}$ is in the upper half of the beam (for the convex bending case).

In bending of a two-layer bimodular laminate since one of the two layers is always in compression (or tension), only three of the four elastic moduli belonging to these layers are pertinent [see Fig. A-2]. This means that the location of the neutral surface depends on only two elastic-moduli ratios. The effects of these two ratios on $h_{1}$ are studied for a wide range of $E_{2}{ }^{c} / E_{2}{ }^{\prime}$ in Table 2. The shear correction coefficient varies drastically with the ratio of the shear moduli of the two layers $\left(G_{1} / G_{2}\right)$, whereas it changes only a little for a wide range of $E_{2}{ }^{c} / E_{2}{ }^{l}$ for $E_{1}{ }^{c} / E_{2}{ }^{t}=1$, as shown in Fig. 1.

The shear correction coefficient for two-layer unimodular laminates increases rapidly from $50 \%$ to $98 \%$ of the classical value $(5 / 6)$ as the elastic-

Table 1. Effect of Bimodular Ratio on Neutral-Surface Location for Single-Layer Bimodular Material.

\begin{tabular}{rc}
\hline Ec/Et & $h_{\mathbf{t}} / \mathbf{h}$ \\
\hline 0 & 0 \\
$1 / 2$ & 0.414 \\
1 & 0.500 \\
2 & 0.586 \\
5 & 0.691 \\
10 & 0.760 \\
100 & 0.909 \\
1000 & 0.969 \\
$\infty$ & 1 \\
\hline
\end{tabular}


Table 2. Effect of Bimodular Ratios on Neutral-Surface Location for Two-Layer Bimodular Laminates $\left(h_{1} / h_{2}=1\right)$.

\begin{tabular}{cccc}
\hline $\mathbf{E}_{2}{ }^{\mathrm{c}} / \mathbf{E}_{2}{ }^{\mathrm{t}}$ & \multicolumn{3}{c}{$\mathbf{h}_{\mathbf{t}} / \mathbf{h}$} \\
\hline & $\mathbf{E}_{1}{ }^{\mathrm{c}} / \mathbf{E}_{2}{ }^{\mathrm{t}}=\mathbf{1 / 2}$ & $\mathbf{E}_{1}{ }^{\mathrm{c}} / \mathbf{E}_{2}{ }^{\mathrm{t}}=\mathbf{1}$ & $\mathbf{E}_{1}{ }^{\mathrm{c}} / \mathbf{E}_{\mathbf{2}}{ }^{\mathrm{t}}=\mathbf{2}$ \\
\cline { 2 - 4 } 0 & 0.411 & 0 & 0.5811 \\
$1 / 4$ & 0.413 & 0.333 & 0.5816 \\
$1 / 2$ & 0.414 & 0.414 & 0.5822 \\
$3 / 4$ & 0.415 & 0.464 & 0.5827 \\
1 & 0.417 & 0.500 & 0.5833 \\
2 & 0.421 & 0.586 & 0.5858 \\
3 & 0.424 & 0.634 & 0.5886 \\
4 & 0.427 & 0.666 & 0.5917 \\
5 & 0.430 & 0.691 & 0.5955 \\
10 & 0.439 & 0.760 & 0.6667 \\
\hline
\end{tabular}

SHEAR-HODUL I RATIO - $G_{1} / G_{2}$

Figure 1. Effect of shear modulus ratio on shear correction coefficient for various bimodulus ratios $\left(E_{2}{ }^{c} / E_{2}{ }^{\prime}\right)$ for two-layer bimodular laminates $\left(h_{1} / h_{2}=1, E_{1}{ }^{\prime} / E_{2}{ }^{\prime}=1 / 2\right)$.

modulus ratio $E_{1} / E_{2}$ varies from 0 to 0.25 as shown in Fig. 2. For ratios greater than 0.5 , a value of $5 / 6$ would be a good approximation for $K^{2}$. The dimensionless maximum shear stress increases from 0 to 12 as $E_{1} / E_{2}$ changes from 0 to infinity as tabulated in Table 3.

Three-layer bimodular laminates which have facings (top and bottom layers) made of the same material are considered here. For the case in which $E_{1}{ }^{c} / E_{2}{ }^{c}=1$, the effect of bimodular ratio $E_{3}{ }^{t} / E_{2}{ }^{c}$ on $h_{t} / h$ for different ratios of $E_{2}{ }^{t} / E_{2}{ }^{c}\left(1 / 2,1\right.$, and 2) are investigated in Table 4. As $E_{3}{ }^{t} / E_{2}{ }^{c}$ increases from 0 to $10, h_{t} / h$ decreases for all three values of $E_{2}{ }^{t} / E_{2}{ }^{c}$. Also, a plot of $K^{2}$ vs $E_{1}{ }^{c} / E_{2}{ }^{c}$ is given in Fig. 3 which shows the shear correction factor as a function of the bimodularity of the material. 


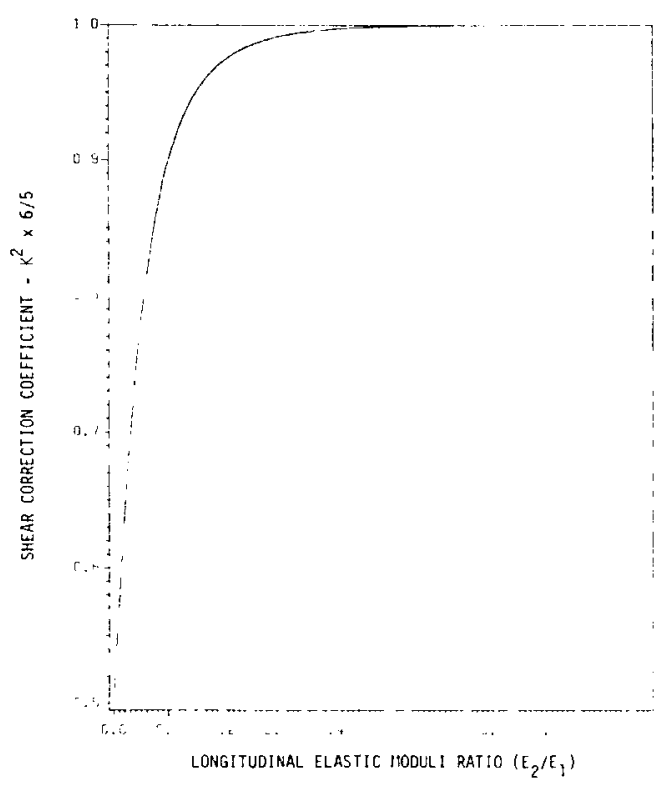

Figure 2. Effect of longitudinal Young's modulus ratio on shear correction coefficient for twolayer unimodular materials $\left(h_{2} / h_{2}=1, G_{1} / G_{2}=1\right)$.

Table 3. Effect of Elastic-Moduli Ratio on Dimensionless Maximum Shear Stress for a Two-Layer Unimodular-Material Laminate $\left(h_{2} / h_{2}=1\right)$.

\begin{tabular}{rc}
\hline$E_{1} / E_{2}$ & $(2 / 3)\left(\tau_{\mathbf{x z}}\right)_{\max }$ \\
\hline 0 & 0 \\
$1 / 4$ & 0.548 \\
$1 / 2$ & 0.727 \\
$3 / 4$ & 0.870 \\
1 & 1.000 \\
2 & 1.456 \\
5 & 2.791 \\
10 & 6.241 \\
100 & 7.087 \\
1000 & 7.897 \\
$\infty$ & 8.000 \\
\hline
\end{tabular}

Finally, the effect of elastic moduli ratio $E_{1} / E_{2}$ on $K^{2}$ and $\left(\tau_{x z}\right)_{\max }$ are studied (see Fig. 4 and Table 5 ) for three-layer unimodular-material laminates with identical facings. For different values of $G_{1} / G_{2}, K^{2}$ increases rapidly for $E_{1} / E_{2}$ less than 0.05 but after this point, $K^{2}$ changes very slowly. The maximum dimensionless shear stress varies from 0 to 40.5 for $0<E_{1} / E_{2}<\infty$. 
Table 4. Effect of Bimodular Ratios on Neutra/-Surface Location for Threo-Layer Bimodular Laminates $\left(h_{1} / h_{2}=h_{3} / h_{2}=1, E_{1}^{c} / E_{2}^{c}=E_{3}^{c} / E_{2}^{c}=1\right)$.

\begin{tabular}{|c|c|c|c|}
\hline$E_{3}{ }^{t} / E_{2}{ }^{c}$ & & $h_{t} / h$ & \\
\hline & $E_{2}{ }^{t} / E_{2}^{c}=1 / 2$ & $E_{2}{ }^{t} / E_{2}{ }^{c}=1$ & $E_{2}{ }^{t} / E_{2}^{c}=2$ \\
\hline 0 & 0.724 & 0.833 & 0.609 \\
\hline $1 / 4$ & 0.643 & 0.722 & 0.573 \\
\hline $1 / 2$ & 0.586 & 0.633 & 0.541 \\
\hline $3 / 4$ & 0.542 & 0.561 & 0.513 \\
\hline 1 & 0.508 & 0.500 & 0.488 \\
\hline 2 & 0.418 & 0.333 & 0.414 \\
\hline 5 & 0.310 & 0.119 & 0.309 \\
\hline 8 & 0.267 & 0.033 & 0.266 \\
\hline 10 & 0.250 & 0 & 0.249 \\
\hline
\end{tabular}

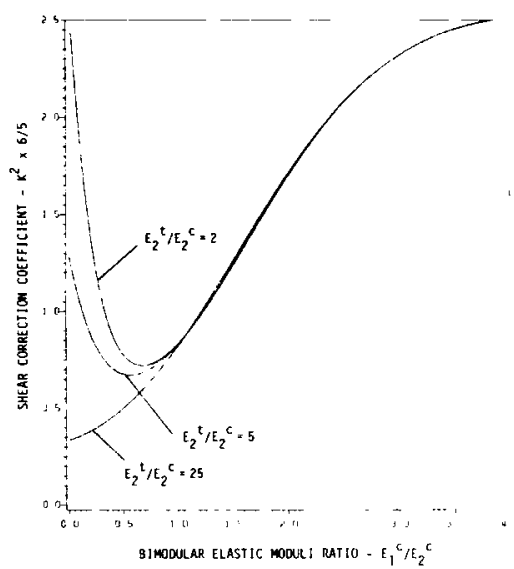

Figure 3. Effect of bimodular elastic moduli ratio $E_{1} / E_{2} C$ on shear correction coefficient for a three-layer bimodular laminate $\left(h_{1} / h_{2}=1, G_{1} / G_{2}=G_{3} / G_{2}=1, E_{1}^{\prime} / E_{2}^{\prime}=2\right)$.

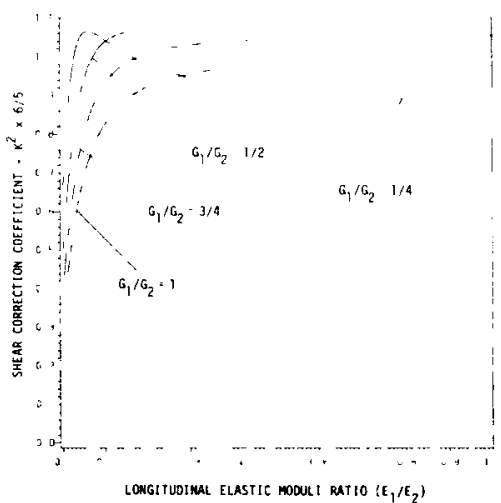

Figure 4. Effect of elastic moduli ratio and shear moduli ratio on shear correction coefficient for three-layer unimodular-material laminates $\left(H_{1} / h_{2}=h_{3} / h_{2}=1, E_{1} / E_{3}=1, G_{1} / G_{3}=1\right)$. 
Table 5. Effect of Elastic-Moduli Ratio on Dimensionless Maximum Shear Stress for a Three-Layer Unimodular-Material Laminate $\left(h_{1} / h_{2}=h_{3} / h_{2}=1, E_{1} / E_{3}=1\right)$.

\begin{tabular}{rc}
\hline$E_{1} / E_{2}$ & $(2 / 3)\left(\bar{\tau}_{\mathbf{x z}}\right)_{\max }$ \\
\hline 0 & 0 \\
$1 / 4$ & 0.257 \\
$1 / 2$ & 0.509 \\
$3 / 4$ & 0.757 \\
1 & 1.000 \\
2 & 1.928 \\
5 & 4.355 \\
10 & 7.500 \\
100 & 21.428 \\
1000 & 26.316 \\
$\infty$ & 27.000 \\
\hline
\end{tabular}

\section{CONCLUSIONS}

Closed-form solutions for the Timoshenko-type shear correction coefficient and the maximum dimensionless transverse shear stress are presented for bimodular laminates undergoing cylindrical bending. These solutions depend on bimodular ratio(s) for different laminates. However, it is interesting to note that both the transverse shear correction coefficient and the maximum dimensionless transverse shear stress $\left(\bar{\tau}_{x z}\right)_{\max }$ for a single-layer material are unaffected by bimodular ratio, $E^{c} / E^{t}$.

For two- and three-layer laminates, both $K^{2}$ and $\left(\bar{\tau}_{x z}\right)_{\max }$ depend upon the bimodular ratios and transverse shear moduli ratios. According to the results presented, in some cases $K^{2}$ is less than the classical value of $5 / 6$.

\section{ACKNOWLEDGMENTS}

The financial support of the Office of Naval Research, Mechanics Division, and the encouragement of Dr. N. L. Basdekas are gratefully acknowledged.

\section{REFERENCES}

1. N. Kamiya, "Transverse Shear Effect in a Bimodulus Plate," Nuclear Engineering and Design, Vol. 32 (1975), p. 351.

2. C. W. Bert and C. J. Rebello, "Bending of Thick Beams Laminated of Bimodular Materials," Engineering Structures, Vol. 5 (1983), p. 227.

3. J. N. Reddy, C. W. Bert, Y. S. Hsu, and V. S. Reddy, "Thermal Bending of Thick Rectangular Plates of Bimodulus Composite Materials," Journal of Mechanical Engineering Science, Vol. 22 (1980), p. 297.

4. C. W. Bert, J. N. Reddy, V. S. Reddy, and W. C. Chao, "Bending of Thick Rectangular Plates Laminated of Bimodulus Composite Materials," AIAA Journal, Vol. 19 (1981), p. 1342. 
5. J. M. Whitney, "Shear Correction Factor for Orthotropic Laminates Under Static Load," ASME Journal of Applied Mechanics, Vol. 40 (1973), p. 302.

6. C. W. Bert, "Simplified Analysis of Static Shear Factors for Beams of Nonhomogeneous Cross Section," Journal of Composite Materials, Vol. 7 (1973), p. 525.

7. T. S. Chow, "On the Propagation of Flexural Waves in an Orthotropic Laminated Plate and its Response to a Impulsive Load," Journal of Composite Materials, Vol. 5 (1971), p. 306.

\section{APPENDIX: DETAILED DERIVATIONS}

In the following, detailed derivations for unimodular and bimodular laminates are given. Three types of lamination are considered: single-layer, two-layer, and three-layer. In the latter two cases, all layers are assumed to be of the same thickness. Also, in the bimodular cases, the effect of bimodularity on elastic-shear moduli is ignored (since for most of materials $\left.G^{t} / G^{\mathcal{c}}=1\right)$. Further, in the bimodular analyses, axial force is assumed to be zero.

\section{Single-Layer* (Bimodular Material)}

Normal and shear stress distributions for single-layer, bimodular material are shown in Fig. A-1. Since the axial force is taken to be zero,

$$
N=\int_{-h_{c}}^{h_{t}} \sigma_{x} d z^{\prime}=x\left[\int_{-h_{c}}^{0} E^{c} z^{\prime} d z^{\prime}+\int_{0}^{h_{t}} E^{t} z^{\prime} d z^{\prime}\right]=0
$$

Using the fact that

$$
h=h_{t}+h_{c}
$$
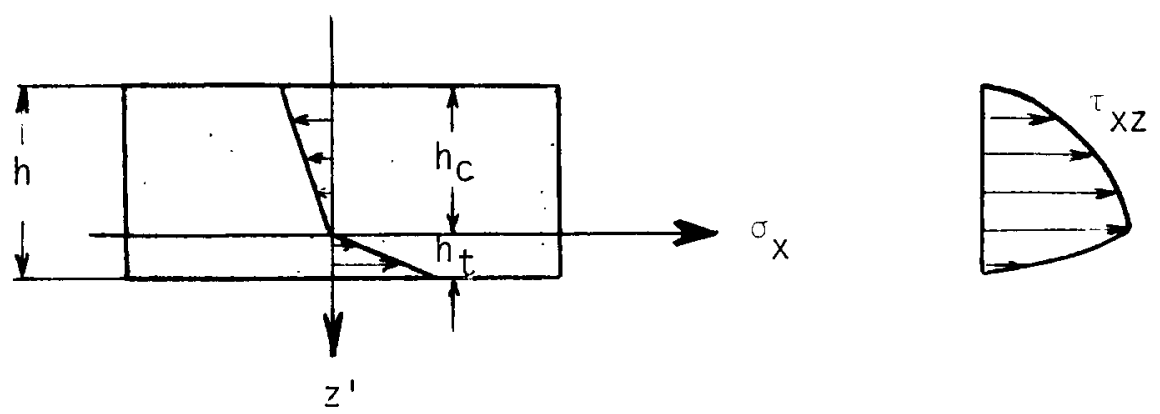

Figure A-1. Normal and shear stress distribution in a single-layer bimodular material.

* This case also might be considered to be a two-layer laminate of ordinary materials but with a certain special thickness ratio $\left(h_{1} / h_{2}=\sqrt{E_{2} / E_{1}}\right)$. 
one can integrate Equation (A-1) to obtain

$$
h_{c} / h=\frac{1}{1+\sqrt{\beta}}, h_{t} / h=\frac{\sqrt{\beta}}{1+\sqrt{\beta}}
$$

where $\beta$ is the bimodular elastic-moduli ratio defined as

$$
\beta=E^{c} / E^{\prime}
$$

From Equations (6) and (9), one has

$$
\begin{gathered}
D=\left(E^{c} h_{c}{ }^{3}+E^{\prime} h_{t}{ }^{3}\right) / 3 \\
b=\frac{1}{2} \begin{cases}E^{c}\left(z^{\prime}\right)^{2}-E^{c} h_{c}^{2} & -h_{c}<z^{\prime}<0 \\
E^{\prime}\left(z^{\prime}\right)^{2}-E^{c} h_{c}^{2} & 0<z^{\prime}<h_{t}\end{cases}
\end{gathered}
$$

Substitution of Equations (A-4) and (A-5) into Equation (15) and using Equation (A-3) leads to

$$
K^{2}=5 / 6
$$

Also, using Equations (19), (20), (A-3), (A-4), and (A-5), one has

$$
\left(\bar{\tau}_{x z}\right)_{\max }=3 / 2
$$

Although the maximum shear stress remains unaffected by bimodularity ratio, the shear-stress distribution does depend on $\beta$. Combining Equations (19), (A-3), (A-4), and (A-5) gives the following shear-stress distribution

$$
\bar{\tau}_{x z}=\frac{3}{2} \begin{cases}\sqrt{\beta}-\sqrt{\beta}(\sqrt{\beta}+1)^{2}(z / h)^{2} ; & -\frac{1}{\sqrt{\beta}+1}<z / h<0 \\ \sqrt{\beta}-\frac{1}{\sqrt{\beta}}(\sqrt{\beta}+1)^{2}(z / h)^{2} ; & 0<z / h<\frac{\sqrt{\beta}}{\sqrt{\beta}+1}\end{cases}
$$

\section{Two-Layer laminates}

(a) Bimodular Material

Each individual layer has different elastic moduli in tension and compression. However, since one layer is only in either a tension or a compression state, only three of the four elastic moduli for two-layer analysis come into the picture (see Fig. A-2). A similar procedure to that used for the single-layer case is used here. To determine the bending and the partial bending-stretching 

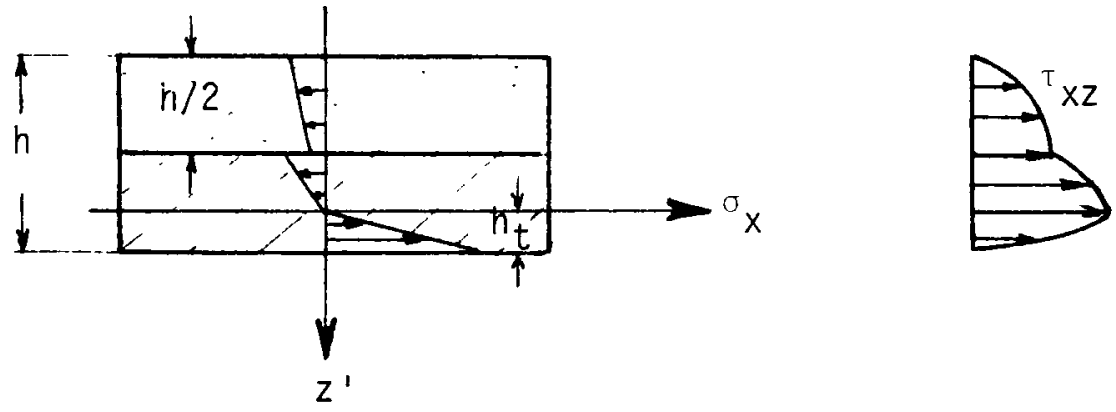

Figure A-2. Normal and shear stress distribution in a two-layer bimodular material laminate.

coupling stiffnesses, one is able to obtain the neutral-surface position from the following quadratic equation

$N=\psi_{\mathrm{k}}\left[\int_{-h_{c}}^{-h_{\mathrm{c}}+h / 2} E_{1}^{{ }^{\prime}} z^{\prime} d z^{\prime}+\int_{-h_{c}+h / 2}^{0} E_{2}{ }^{c} z^{\prime} d z^{\prime}+\int_{0}^{h_{1}} E_{2}{ }^{\prime} z^{\prime} d z^{\prime}\right]=0$

Knowing the neutral-surface location, one can easily determine the following stiffnesses

$$
D=\left\{E _ { 1 } { } ^ { c } \left[\left(h / 2-h_{\partial^{3}}+h_{c}{ }^{3}\right]+E_{2}{ }^{c}\left(h / 2-h{\partial^{3}}^{3}+E_{2}{ }^{t} h_{t}{ }^{3}\right\} / 3\right.\right.
$$

and

$$
b=\frac{1}{2}\left\{\begin{array}{lc}
E_{1}{ }^{c}\left(z^{\prime}\right)^{2}-E_{1}{ }^{c} h_{c}{ }^{2} & -h_{c}<z^{\prime}<-h_{c}+h / 2 \\
E_{2}{ }^{c}\left(z^{\prime}\right)^{2}-E^{2} h_{t}{ }^{2} & -h_{c}+h / 2<z^{\prime}<0 \\
E_{2}{ }^{t}\left(z^{\prime}\right)^{2}-E_{2}{ }^{t} h_{t}{ }^{2} & 0<z^{\prime}<h_{t}
\end{array}\right.
$$

Substituting Equations (A-10) and (A-11) into Equation (15), one has

$$
\begin{gathered}
K^{2}=\frac{5}{6} \cdot \frac{16 g_{1} /(\alpha+\beta)\left(h / 2-h_{\partial^{3}}+\alpha h_{c}^{3}+h_{l}^{3}\right]^{2}}{h\left(g_{1}+1\right)\left[3 ( g _ { 1 } \alpha ^ { 2 } - \beta ^ { 2 } ) \left(h / 2-h_{\partial^{5}}-10\left(g_{1} \alpha^{2} h_{c}^{2}-\beta h^{2}\right)\left(h / 2-h_{\partial^{3}}\right.\right.\right.} \\
\frac{\left.15\left(g_{1} \alpha^{2} h_{c}^{4}-h_{l}^{4}\right)\left(h / 2-h_{c}\right)+8\left(g_{1} \alpha^{2} h_{c}^{5}+h_{l}^{5}\right)\right]}{\text { (A- }}
\end{gathered}
$$

where

$$
\alpha=E_{1}{ }^{c} / E_{2}{ }^{t}, \beta=E_{2}{ }^{c} / E_{2}{ }^{t} \text { and } g_{1}=G_{1} / G_{2}
$$

Since $h_{c} / h$ and $h_{t} / h$ depend upon $\alpha$ and $\beta, K^{2}$ is a function of $g_{1}, \alpha$, and $\beta$ for 
the case of equal-thickness layers. The maximum dimensionless shear stress also is a function of the bimodular ratios $(\alpha, \beta)$

$\left(\bar{\tau}_{x z}\right)_{\text {max }}=\frac{3}{2} \cdot \frac{h}{\alpha\left[\left(h / 2-h_{\delta^{3}}+h_{d}^{3}+\beta\left(h / 2-h_{d^{3}}+h_{t}^{3}\right.\right.\right.} \begin{cases}\alpha\left[h_{c}^{2}-\left(h / 2-h_{d^{2}}\right]\right. & -h_{c}<z^{\prime}<-h_{c}+h / 2 \\ h_{t}{ }^{2} & 0<z^{\prime}<h_{t}\end{cases}$

(b) Unimodular Material

A similar lamination as used in part (a) is considered here. The normal and shear stress distributions are shown in Fig. A-3. Since the flexural-extensional coupling stiffness (b) does not vanish, one must use Equation (13) in order to compute $K^{2}$. Let the transverse shear moduli be the same for both layers $\left(G^{2} / G^{1}=1\right)^{*}$ and $\alpha=E^{2} / E^{1}$. Then, the stiffnesses can be expressed as

$$
\begin{aligned}
& A=E_{1} h(\alpha+1) / 2 \\
& B=E_{1} h^{2}(\alpha-1) / 8 \\
& D=E_{1} h^{3}(\alpha+1) / 24
\end{aligned}
$$

and

$$
(a, b)=E_{1}\left\{\begin{array}{lc}
{\left[(z+h / 2),\left(z^{2}-h^{2} / 4\right) / 2\right]} & -h / 2<z<0 \\
{\left[(\alpha z+h / 2),\left(\alpha z-h^{2} / 4\right) / 2\right]} & 0<z<h / 2
\end{array}\right.
$$

Substitution of Equations (A-14) and (A-15) into Equation (13) gives

$$
K^{2}=\frac{5}{6} \cdot \frac{\left(\alpha^{2}+14 \alpha+1\right)^{2}}{2\left[\alpha^{4}+12 \alpha^{3}+102 \alpha^{2}+12 \alpha+1\right]}
$$
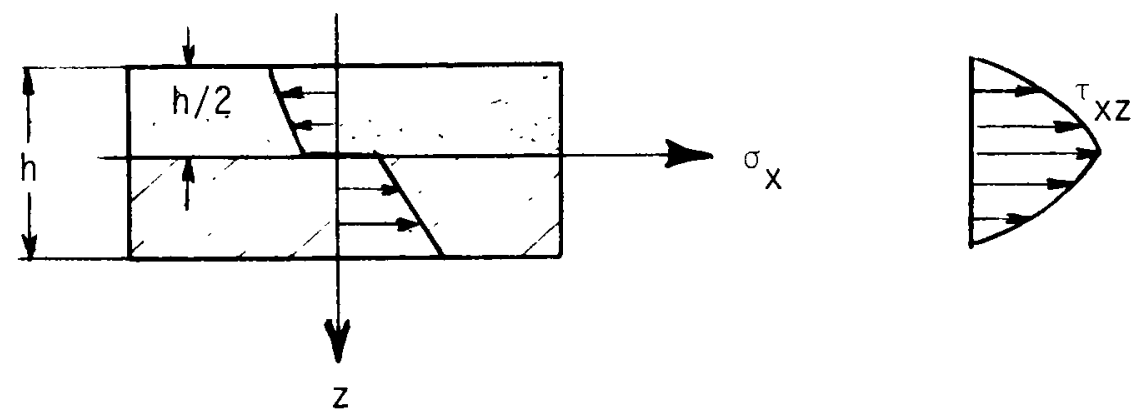

Figure A-3. Normal and shear stress distribution in a two-layer unimodular material laminate.

*This case also might be considered to be a two-layer laminate of ordinary materials but with a certain special thickness ratio $\left(h_{1} / h_{2}=\sqrt{E_{2} / E_{1}}\right)$. 
It is interesting to note that $K^{2}$ as given by Equation (A-16) gives the same result for $\alpha=c$ and $\alpha=1 / c$, where $c$ is an arbitrary constant. Combining Equations (4), (12), (19), (A-14), and (A-15) one gets

$$
\left(\bar{\tau}_{x z}\right)_{\max }=\frac{3}{2} \cdot \frac{8 \alpha(\alpha+1)}{\alpha^{2}+14 \alpha+1}
$$

which shows that $\left(\bar{\tau}_{x z}\right)_{\max }$ depends on elastic-moduli ratio $\left(E_{2} / E_{\downarrow}\right)$, only.

\section{Three-Layer Laminate}

(a) Bimodular Material

Consider a three-layer laminate with the top and bottom layers (facings) made of the same material (i.e., $E_{1}{ }^{c}=E_{3}{ }^{c}, E_{1}{ }^{t}=E_{3}{ }^{t}$ ) as shown in Fig. A-4 in the convex downward bending position. One assumes that the neutral-surface position is within the middle layer (which happens for most practical cases). The axial force vanishes if

$$
\int_{-h_{c}}^{-h_{c}+h / 3} E_{1}{ }^{c} z^{\prime} d z^{\prime}+\int_{-h_{c}+h / 3}^{0} E_{2} z^{\prime} d z^{\prime}+\int_{0}^{h_{t}-h / 3} E_{2}^{\prime} z^{\prime} d z^{\prime}+\int_{h_{t}-h / 3}^{h_{t}} E_{1} z^{\prime} d z^{\prime}=0
$$

Equation (A-18) can be used to determine the neutral-surface position. Having the values of $h_{t}$ and $h_{c}$ enables one to calculate $K^{2}$ with the same procedure as used for the two-layer case

$$
D=\frac{E_{2}{ }^{c}}{3}\left\{\alpha \left[h_{c}^{3}+\left(h / 3-h d^{3}\right]-\left(h / 3-h d^{3}+\beta\left(h_{t}-h / 3\right)^{3}+\gamma\left[h_{t}^{3}-\left(h_{t}-h / 3\right)^{3}\right]\right\}\right.\right.
$$
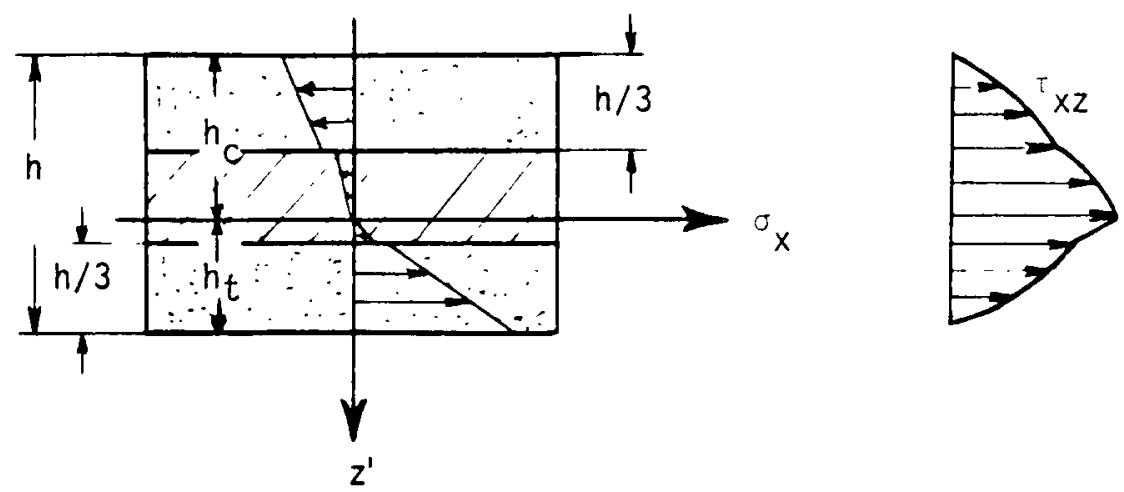

Figure A-4. Normal and shear stress distribution in a three-layer bimodular material laminate. 


$$
\begin{aligned}
& \int_{-h_{c}}^{h_{t}} G^{(k)} d z=G_{2} h\left[2 g_{1}+1\right] / 3
\end{aligned}
$$

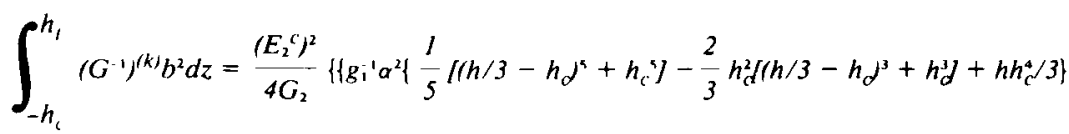

$$
\begin{aligned}
& -\left[\frac { l } { 5 } \left(h / 3-h_{d^{5}}+\frac{2}{3} H_{d}^{2}\left(h / 3-h_{d^{3}}+\left(H_{d}^{2}\right)^{2}\left(h / 3-h_{d}\right)\right.\right.\right. \\
& +\left[\frac{\beta^{2}}{5}\left(h_{t}-h / 3\right)^{5}+\frac{2 \beta}{3} H_{d}^{2}\left(h_{t}-h / 3\right)^{3}+\left(H_{d^{2}}^{2}\left(h_{t}-h / 3\right)\right]\right. \\
& \left.\left.\left.+g_{1}^{-1} \gamma^{2}\left\{\frac{l}{5}\left[h_{t}^{9}-\left(h_{t}-h / 3\right)^{3}\right\}-\frac{2}{3} h_{f}^{2} h_{t}^{3}-\left(h_{t}-h / 3\right)^{3}\right\}+h h_{t}^{4 / 3}\right\}\right\}\right\}
\end{aligned}
$$

where

$$
\begin{gathered}
H_{c}{ }^{2}=\left(h / 3-h^{2}{ }^{2}(\alpha-1)-h_{c}{ }^{2}\right. \\
\alpha=E_{1}{ }^{c} / E_{2}{ }^{c}, \beta=E_{1}{ }^{\prime} / E_{2}{ }^{c}, \gamma=E_{3}{ }^{t} / E_{2}{ }^{c} \text {, and } g_{1}=G_{1} / G_{2}
\end{gathered}
$$

By substituting Equations (A-19), (A-20), and (A-21) into Equation (15), one can determine $K^{2}$. Also, using Equations (A-19 and the following expression for $b_{\max }$ (the maximum value of partial coupling stiffness $b$ ) in the respective regions, one has the maximum dimensionless shear stress by Equation (19).

$$
b_{\max }=\frac{1}{2}\left\{\begin{array}{lc}
L_{1} E_{1}{ }^{c} & -h_{c}<z^{\prime}<-h_{c}+\mathrm{h} / 3 \\
L_{1} E_{1}{ }^{c}-L_{2} E_{2}{ }^{c} & -h_{c}+h / 3<z^{\prime}<0 \\
L_{4} E_{1}{ }^{c}-L_{2} E_{2}{ }^{c}+L_{3} E_{2}{ }^{l} & 0<z^{\prime}<h_{t}-h / 3 \\
L_{4} E_{1}{ }^{t} & h_{t}-h / 3<z^{\prime}<h_{t}
\end{array}\right.
$$

where

$$
\begin{array}{ll}
L_{1}=\left(h-h_{c}\right)^{2}-h_{c}{ }^{2} & L_{3}=\left(h-h_{t}\right)^{2} \\
L_{2}=L_{1}+h_{c}{ }^{2} & L_{4}=L_{3}-h_{t}^{2}
\end{array}
$$

(b) Unimodular Material

Shear, bending, and partial stretching-bending stiffnesses for the threelayer symmetric laminate* with equal-thickness layers as shown in Fig. A-5 are $\left(g_{1}=G_{1} / G_{2}, \alpha=E_{1} / E_{2}\right)$

*The top and bottom layers have the same properties. 

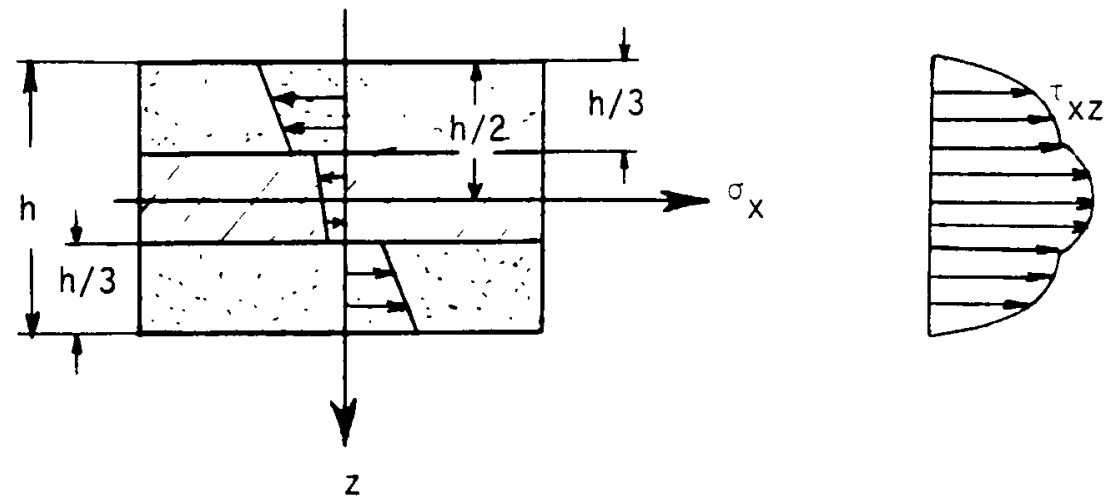

Figure A-5. Normal and shear stress distribution in a three-layer unimodular material laminate.

$$
\begin{aligned}
& S=\frac{G_{2} h}{3}\left(1+2 g_{1}\right), \\
& D=\frac{E_{2} h^{3}}{324}(26 \alpha+1),
\end{aligned}
$$

and

$$
\int_{-h / 2}^{h / 2}\left(G^{-1}\right)^{(k)} b^{2} d z^{\prime}=\frac{E_{2}{ }^{2} h^{5}}{29160}\left[102 \alpha^{2}+g_{1}\left(120 \alpha^{2}+20 \alpha+\right)\right]
$$

Substituting these stiffnesses into Equation (13) leads to

$$
K^{2}=\frac{5}{6} \cdot \frac{\left[26_{\alpha}+1\right]^{2} g_{1}}{\left(1+2 g_{1}\right)\left[102 \alpha^{2}+g_{1}\left(120 \alpha^{2}+20 \alpha+1\right)\right]}
$$

Absolute dimensionless maximum shear stress occurs at the midplane. Using the same procedure as used before, one obtains

$$
\left(\bar{\tau}_{x z}\right)_{\max }=\left(\bar{\tau}_{x z}\right)_{z=0}=\frac{3}{2} \cdot \frac{3(8 \alpha+1)}{26 \alpha+1}
$$

and the shear stress at the interfaces is

$$
\left(\tau_{x z}\right)_{z= \pm h / 6}=\frac{3}{2} \quad \frac{24 \alpha}{26 \alpha+1}
$$

It is interesting to note that 


$$
\frac{\left(\bar{\tau}_{x z}\right)_{\max }}{\left(\bar{\tau}_{x z}\right)_{z= \pm h / 6}}=I+(1 / 8 \alpha)
$$

\section{SYMBOLS}

\begin{tabular}{|c|c|}
\hline a & partial extensional stiffness \\
\hline A & extensional stiffness \\
\hline b & partial flexural-extensional coupling stiffness \\
\hline B & flexural-extensional coupling stiffness \\
\hline c & arbitrary constant \\
\hline $\mathrm{D}$ & flexural stiffness \\
\hline $\mathrm{E}$ & longitudinal Young's modulus \\
\hline g & transverse shear moduli ratio \\
\hline G & transverse shear moduli \\
\hline h & beam thickness \\
\hline $\mathrm{H}_{\mathrm{c}}$ & defined by Equation (A-22) \\
\hline $\mathrm{K}^{2}$ & shear correction coefficient \\
\hline $\mathrm{L}_{\mathrm{i}}(\mathrm{i}=1,2,3,4)$ & defined by Equation (A-24) \\
\hline $\mathrm{M}$ & moment resultant \\
\hline $\mathbf{N}$ & force resultant \\
\hline Q & transverse shear resultant \\
\hline S & thickness-shear stiffness \\
\hline $\mathbf{u}, \mathbf{w}$ & displacements in $\mathrm{x}$ and $\mathrm{z}$ directions \\
\hline $\mathrm{x}$ & coordinate along the length \\
\hline $\mathbf{z}$ & $\begin{array}{l}\text { thickness-direction coordinate, measured positive downward } \\
\text { from the midplane }\end{array}$ \\
\hline $\mathbf{z}^{\prime}$ & $\begin{array}{l}\text { thickness-direction coordinate, measured positive } \\
\text { downward from neutral-surface position }\end{array}$ \\
\hline$z_{n}$ & $\begin{array}{l}\text { neutral-surface location for zero normal strain in the } \mathrm{x} \\
\text { direction }\end{array}$ \\
\hline$\alpha, \beta, \gamma$ & bimodular longitudinal Young's moduli ratios \\
\hline$\varepsilon_{\mathrm{x}}$ & normal strain in the $\mathrm{x}$ direction \\
\hline$\gamma_{x z}, \bar{\gamma}_{x z}$ & $\begin{array}{l}\text { engineering shear strain in } \mathrm{xz} \text { plane: actual and uniform- } \\
\text { shear-distribution cases }\end{array}$ \\
\hline$\tau_{x z}$ & shear stress in $\mathrm{xz}$ plane \\
\hline$\sigma_{\mathrm{x}}$ & normal stress in the $\mathrm{x}$ direction \\
\hline$\psi$ & bending slope \\
\hline \multicolumn{2}{|l|}{ Subscript } \\
\hline $1,2,3$ & layer number \\
\hline()$, x$ & $\partial() / \partial x$ \\
\hline \multicolumn{2}{|l|}{ Superscript } \\
\hline 0 & midplane \\
\hline- & dimensionless \\
\hline$(c, t)$ & (compression, tension) \\
\hline (k) & index $(=c, t)$ \\
\hline
\end{tabular}

